\title{
Energy-recycling high-contrast organic light-emitting devices
}

\author{
Chih-Jen Yang \\ Ting-Yi Cho \\ Chun-Liang Lin \\ Chung-Chih Wu
}

\begin{abstract}
It is reported that by integrating OLEDs with solar cells, ambient-light reflection as low as $1.4 \%$ (even superior to that achieved with polarizers) can be achieved without compromising the EL efficiency for high-contrast display applications. Furthermore, in such a configuration, the photon energies of both the incident ambient light and the portion of OLED emission not getting outside of the device can be recycled into useful electrical power via the photovoltaic action, instead of being wasted as in other reported contrast-enhancement techniques. These features, we believe, shall make this present technique attractive for high-contrast display applications and portable/mobile electronics that are highly power-aware.
\end{abstract}

Keywords - OLED, solar cell, high contrast, energy recycling.
Organic light-emitting devices (OLEDs), either conventional bottom-emitting or top-emitting, in general are composed of a reflective back electrode, organic layers, and a (semi-) transparent exit electrode for light out-coupling. ${ }^{1-3}$ With the reflective back electrode, OLEDs in general exhibit rather strong reflection. The contrast ratio of a display is defined as the ratio of its brightness at the bright (on) state and its brightness at the dark (off) state. Hence, such reflection would seriously degrade the contrast of an OLED display under a strong lighting environment, since even at the off-state, the device will still exhibit some brightness due to reflection of the ambient illumination. Polarizer or filter films may be laminated on the surface of the display panel to reduce reflection of ambient illumination. ${ }^{4}$ However, the use of such contrast-enhancement films reduces the power efficiency of the displays below $\sim 40 \%$ of the device efficiency due to their strong absorption and also adds extra complexity and cost in fabrication. OLED structures that could integrate the characteristics of low ambientlight reflection within the device structure and yet retain as much emission efficiency will thus be particularly useful, since the display contrast could then be enhanced with reduced impacts on cost and efficiency.

Several device structures have been reported for reducing reflection and for improving contrast. ${ }^{4-9}$ All these structures involve inserting extra layer(s) of optical purposes (absorption, interference or both) into the active region of devices (i.e., between the anode and the cathode). Specifically, these optical layers are inserted neighboring to the reflective metal cathode. For current conduction, such layers must also have appropriate electrical characteristics (i.e., carrier injection and transport). However, the high-contrast OLED structures above all deal with bottom-emitting OLEDs. Recently, we reported a high-contrast top-emitting OLED. ${ }^{10}$ Unlike previous high-contrast devices that all involve inserting extra layer(s) of optical purposes into the active region of devices, our device utilizes only optical characteristics of electrodes and anti-reflection (AR) coatings deposited outside the active region of the device, thus reducing impacts on electrical characteristics and device complexity.

For all these contrast-enhancement approaches, the incident photon energies are simply absorbed and wasted. Moreover, in these contrast-enhancement structures, a significant portion of the internal OLED emission cannot come out and is thus also wasted. Such wasted photon energies may be recycled by placing a solar cell in the back of an OLED. With such a structure, the incident ambient light in principle can be absorbed by the solar cell, thus reducing the reflection and giving higher contrast. Yet, in contrast with previous contrast-enhancement approaches, the absorbed ambient light can now be converted to useful electrical power, instead of being wasted. The portion of internal OLED emission that cannot come out shall also be similarly absorbed by the solar cell and be converted to useful electrical power. Such a feature of energy recycling may be of significance and use for portable/mobile electronics, since such devices typically are highly power-aware. With such power recycling, the devices themselves would have the added self-powering function and the higher system-level power efficiency. Both could well contribute to longer battery lifetimes of portable/mobile electronics. In this paper, we report such integration of OLEDs and solar cells, which not only exhibits a contrast superior to the conventional polarizer approach but also is capable of recycling both incident ambient illumination and internally generated OLED emission.

The device structure studied in this work is shown in Fig. 1, which consists of an inverted top-emitting OLED stacked back to back on top of an organic solar cell. A metal (e.g., Mo) in combination with indium tin oxide (ITO, 40 $\mathrm{nm}$ ) was used as the bottom anode of the solar cell. Both

Extended revised version of a paper presented at the 2007 International Display Manufacturing Conference (IDMC '07) held July 3-6, 2007, in Taipai, Taiwan. The authors are with the Department of Electrical Engineering, Graduate Institute of Electro-Optical Engineering and Graduate Institute of Electronics Engineering, National Taiwan University, Taipei, Taiwan 10617, R.O.C.; telephone +886-932-142-660, fax +886-2-2368-9178, e-mail: chungwu@cc.ee.ntu.edu.tw.

(C) Copyright 2008 Society for Information Display 1071-0922/08/1606-0691\$1.00 


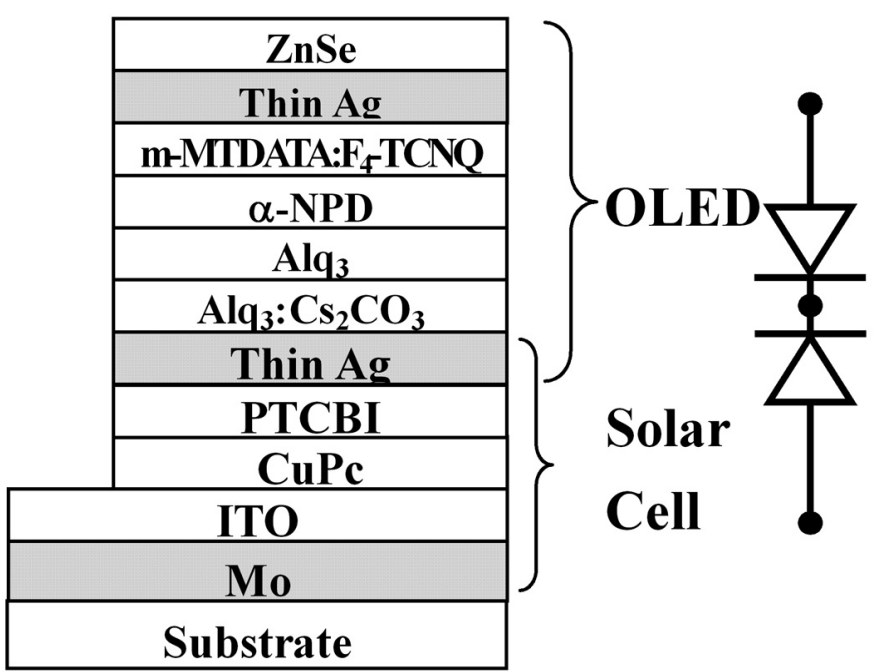

FIGURE 1 - The structure of the [OLED plus solar cell] stack.

ITO and Mo were deposited by magnetron sputtering. The organic solar cell had the conventional single donor-acceptor (D-A) heterostructure. ${ }^{11,12}$ Copper phthalocyanine $(\mathrm{CuPc}, 30 \mathrm{~nm})$ was used as the donor and 3,4,9,10-perylenetetracarboxylic bis-benzimidazole (PTCBI, $50 \mathrm{~nm}$ ) was used as the acceptor. ${ }^{11,12} \mathrm{~A}$ thin layer $(18 \mathrm{~nm})$ of $\mathrm{Ag}$ was deposited on top of the D-A stack as the common semitransparent cathode for both the solar cell and the inverted OLED.

The organic multilayers of the inverted top-emitting OLED in sequence consisted of tris-(8-hydroxyquinoline) aluminum ( $\mathrm{Alq}_{3}, 10 \mathrm{~nm}$ ) doped with 20 wt. $\%$ of $\mathrm{Cs}_{2} \mathrm{CO}_{3}$ as the electron-injection layer, ${ }^{1,13,14}$ intrinsic $\mathrm{Alq}_{3}(50 \mathrm{~nm})$ as the electron-transport and emitting layer, and $\alpha$-naphthylphenylbiphenyl diamine $(\alpha-\mathrm{NPD}, 20 \mathrm{~nm})$ as the hole-transport layer, 4,4',4"-tris(3- methylphenylphenylamino)triphenylamine ( $m$-MTDATA, $30 \mathrm{~nm})$ doped with $2 \mathrm{wt} . \%$ of tetrafluorotetracyano-quinodimethane $\left(\mathrm{F}_{4}\right.$-TCNQ) as the hole-injection layer. ${ }^{15}$ Finally, a thin layer $(20 \mathrm{~nm})$ of Ag was deposited on top as the semi-transparent anode of the inverted OLED. The thin layer of Ag with the thickness of 18-20 nm has relatively low optical absorption and high conductivity. The top Ag anode of OLED was further capped with a high-index dielectric layer of thermally evaporated ZnSe $(n \sim 2.4-2.5,30 \mathrm{~nm})$ as an index-matching layer to fine tune optical properties of the top electrode and the complete device stack. ${ }^{16,17}$ The thicknesses of the transparent ITO layer, organic layers, and the ZnSe layer had been carefully designed to optimize colors and efficiencies of the OLED and the reflection properties of the complete device stack. All the organic layers and the metal electrode layers were deposited in sequence by thermal evaporation without breaking the vacuum. After the device deposition, the devices were encapsulated with a cover glass under a dry nitrogen atmosphere. The conventional bottom-emitting OLED with the structure of ITO anode/m-MTDATA $(30 \mathrm{~nm}) / \alpha-\mathrm{NPD}(20 \mathrm{~nm}) / \mathrm{Alq}_{3}(60 \mathrm{~nm}) / \mathrm{LiF}(0.5 \mathrm{~nm}) / \mathrm{Al}$

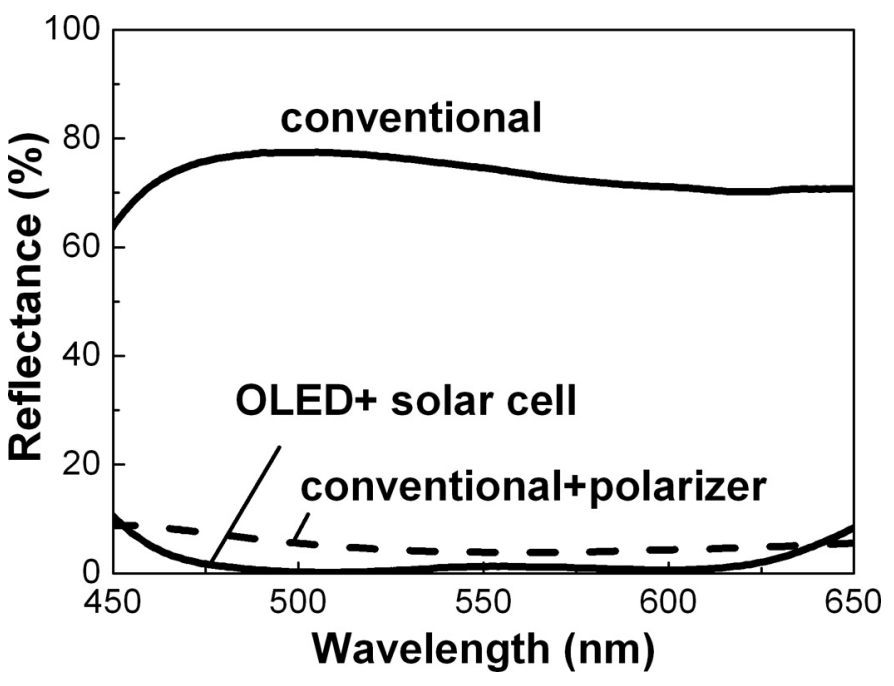

FIGURE 2 - Measured reflectance spectra of the [OLED plus solar cell] stack, and the conventional bottom-emitting OLEDs with and without lamination with a circular polarizer film.

cathode was also fabricated and tested in this study for comparison purposes.

Figure 2 shows the measured reflectance spectra of a conventional bottom-emitting device with or without lamination with a circular polarizer and the integrated [topemitting OLED plus solar cell] stack. The reflectance of the conventional OLED is high (up to $80 \%$ ) over the visible range, while that of the [OLED plus solar cell] stack remains low over the whole visible range (even lower than that of a conventional OLED plus a polarizer over most of the visible range). The luminous reflectance $R_{L}$, the effective reflectance to human eyes under a light source with a spectral power distribution $S(\lambda)$, is defined as $R_{L}=\int V(\lambda) S(\lambda) R(\lambda)$ $d \lambda / V(\lambda) S(\lambda) d \lambda$, where $V(\lambda)$ is the standard photopic curve and $R(\lambda)$ is the reflectance spectrum. Here using $S(\lambda)$ of the standard illuminating source D65, one obtains a luminous reflectance of $\sim 1.4 \%$ for the the [OLED plus solar cell] stack, which is nearly two orders of magnitude lower than that $(70.6 \%)$ of the bottom-emitting device. Indeed, such a

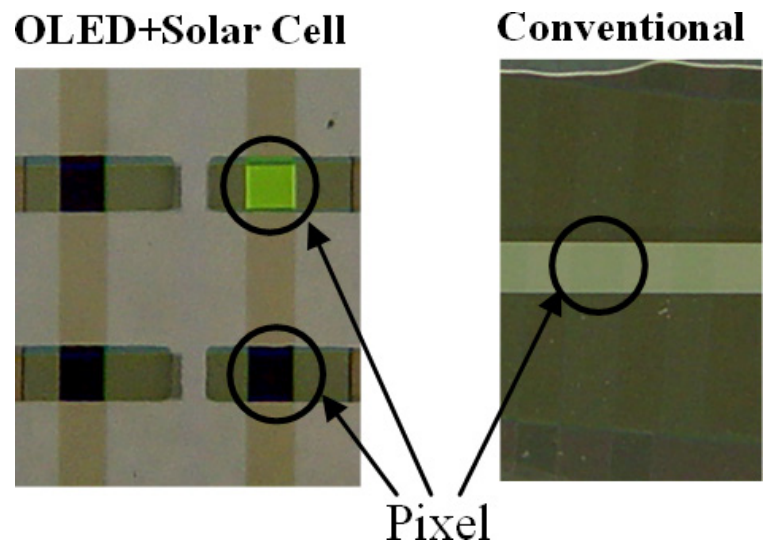

FIGURE 3 - Color photos of the [OLED plus solar cell] stack and the conventional bottom-emitting OLED. 


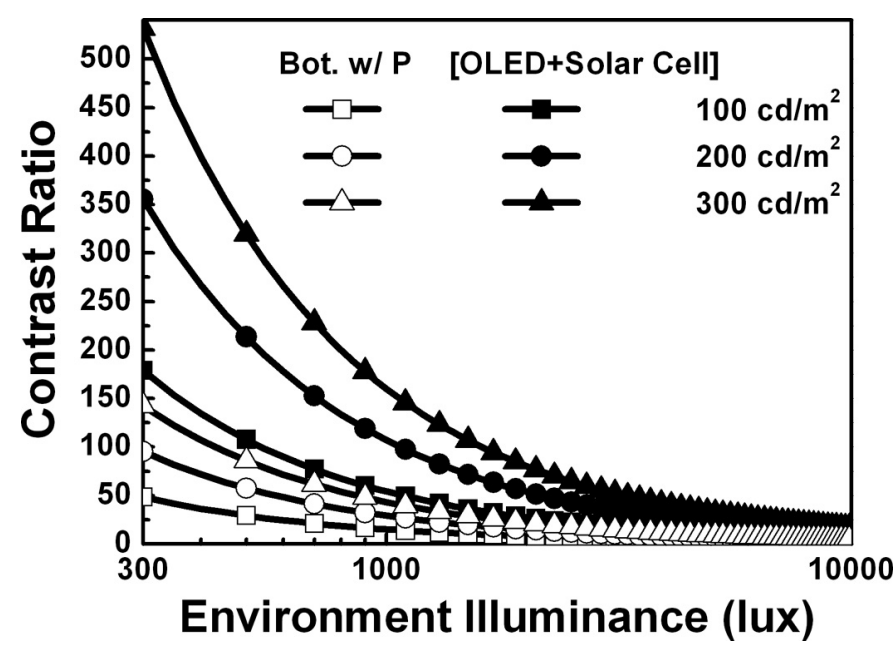

FIGURE 4 - Contrast ratio vs. environment illuminance of the energy-recycling OLED and the conventional bottom-emitting OLED with a polarizer film.

luminous reflectance is even superior to that $(\sim 5-6 \%)$ of the conventional OLED laminated with the polarizer. Photos in Fig. 3 show the appearance of a low-reflection [OLED + solar cell] stack (with one OLED on and others off) and a highly reflective bottom-emitting OLED under strong ambient illumination. For the present [OLED plus solar cell] stack, the high contrast between the off pixel (which is almost completely black) and the on pixel is clearly seen without using any contrast- enhancement films.

Based on the results shown in Fig. 2, we further calculate the display contrast ratios of the devices. Figure 4 shows the contrast ratio as the function of environment illuminance at different device brightnesses. The contrast ratio of the present [OLED plus solar cell] stack is higher than that of the conventional bottom-emitting OLED laminated with

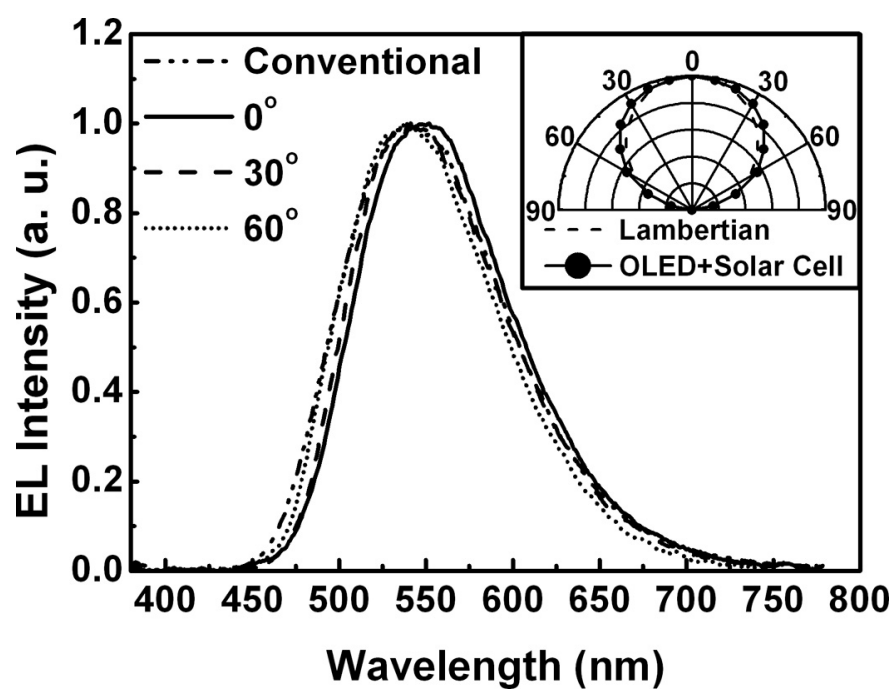

FIGURE 5 - EL spectra of the energy-recycling OLED at $0^{\circ}, 30^{\circ}$, and $60^{\circ}$ off the surface normal, along with the EL spectrum of the bottom-emitting OLED at $0^{\circ}$. Inset: the polar plot of the emission intensity of the energy-recycling OLED compared to the Lambertian pattern.

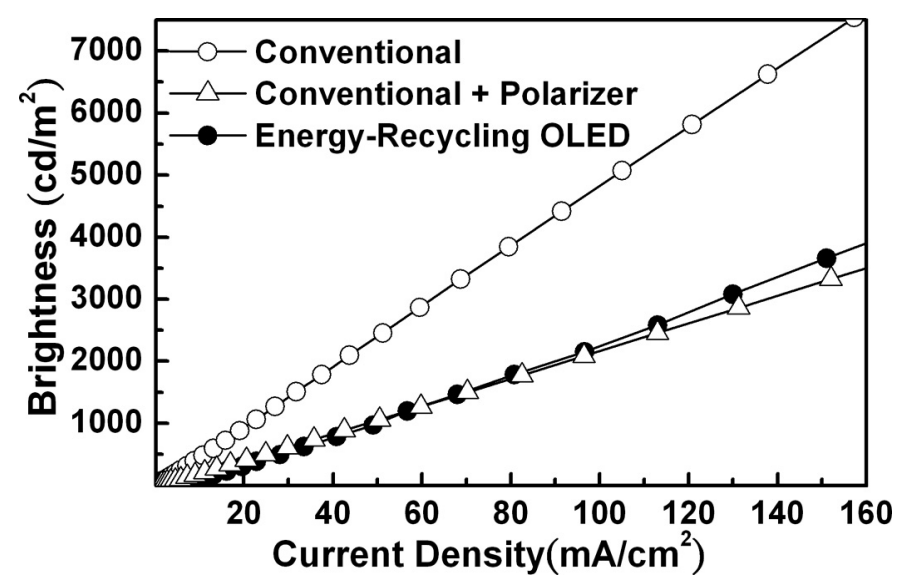

FIGURE $6-L-I$ characteristics of the energy-recycling OLED, and the conventional bottom-emitting OLED with or without lamination with a polarizer film.

a circular polarizer. At the device brightness of $200 \mathrm{~cd} / \mathrm{m}^{2}$ and an environment illuminance of 1000 lux, the [OLED plus solar cell] stack gives over 3 times higher contrast ratio (107.3 vs. 29.4) than the bottom-emitting OLED laminated with a circular polarizer.

Figure 5 shows the EL spectra measured at viewing angles of $0^{\circ}, 30^{\circ}$, and $60^{\circ}$ off the surface normal for the low-reflection energy-recycling top-emitting OLED and that at $0^{\circ}$ for the conventional OLED. The emission spectra of the energy-recycling OLED are nearly the same as that of the conventional device and show hardly any shift with viewing angles. Furthermore, the measured emission pattern of the energy-recycling OLED is close to the ideal Lambertian, as shown in the inset of Fig. 5. Overall, through introducing the absorptive materials beneath the OLED (via the solar cell) and through tuning the layer thicknesses in the structure, the present [OLED plus solar cell] stack achieves the very broad low-reflection band and stable emission colors/brightness with viewing angles.

Figure 6 compares the brightness-current characteristics of the conventional bottom-emitting device and the low-reflection energy-recycling top-emitting device. The top-emitting device exhibits an efficiency of $\sim 2.5 \mathrm{~cd} / \mathrm{A}$, which is about $50 \%$ of the cd/A efficiency of the conventional device $(\sim 5 \mathrm{~cd} / \mathrm{A})$ and is slightly higher than that of the conventional OLED laminated with the circular polarizer $(\sim 2.1 \mathrm{~cd} / \mathrm{A})$.

Figure 7(a) shows the $I-V$ characteristics of the solar cell (beneath the OLED) both in the dark and under the AM 1.5 illumination. In the inset of Fig. 7(a), the spectral response (external quantum efficiency as a function of the wavelength) of the device under the external illumination is shown. The solar cell shows a peak quantum efficiency of $\sim 6 \%$ around $600 \mathrm{~nm}$. The dark current (dashed line) of the solar cell follows well-behaved diode characteristics with a rectification ratio of $\sim 1 \times 10^{5}$ at $\pm 1 \mathrm{~V}$. As the device is illuminated at one sun with the AM 1.5 solar simulator, the solar cell gives a short-circuit current $\left(I_{\mathrm{sc}}\right)$ of $1.35 \mathrm{~mA} / \mathrm{cm}^{2}$ and an open-circuit voltage $\left(V_{\text {oc }}\right)$ of $0.4 \mathrm{~V}$. With the filling factor 
(a)
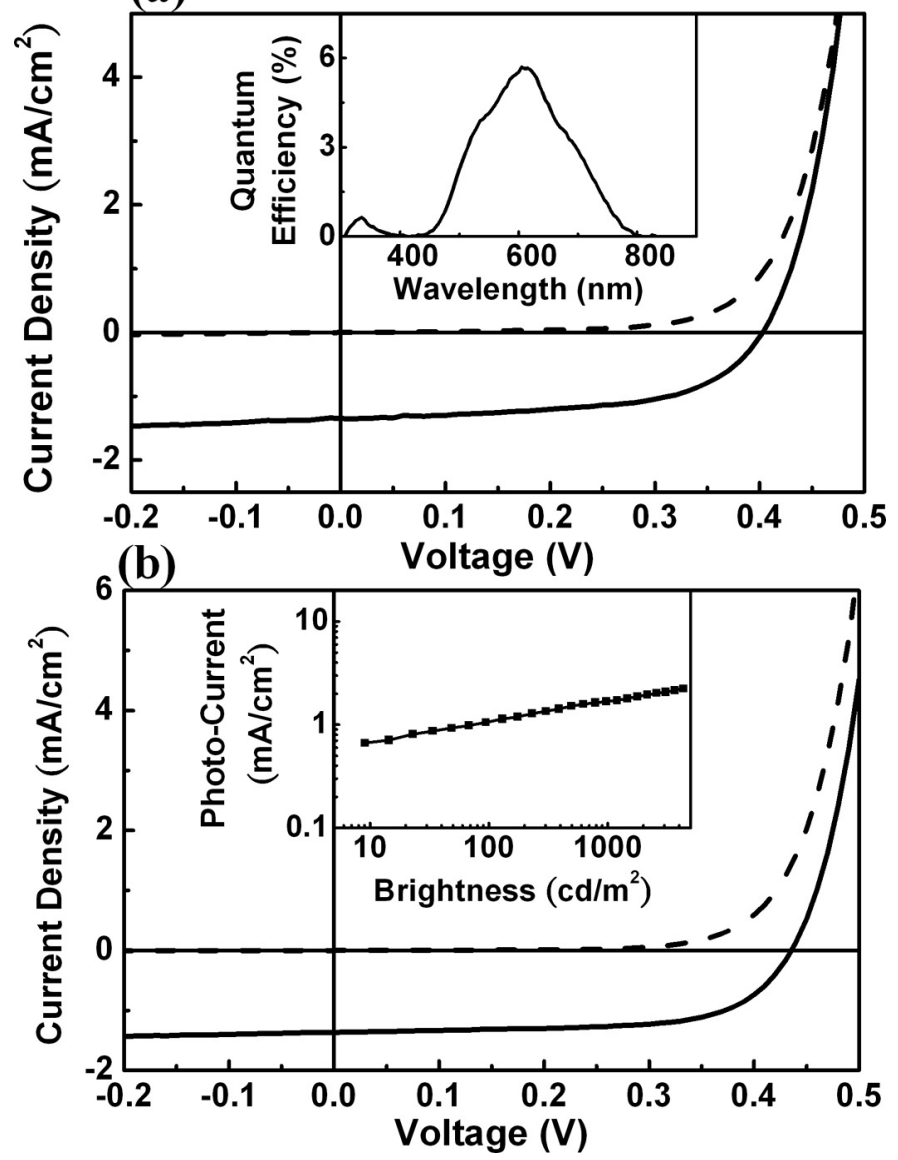

FIGURE $7-$ (a) $I-V$ characteristics of the solar cell in the dark and under the AM 1.5 illumination. Inset of (a): Spectral response of the cell under external illumination. (b) $I-V$ characteristics of the solar cell in dark and under the illumination of the OLED above (driven at $20-\mathrm{mA} / \mathrm{cm}^{2}$ $315-\mathrm{cd} / \mathrm{m}^{2}$ external brightness). Inset of (b): Photocurrent of the solar cell vs. external brightness of the OLED.

(FF) of $58 \%$, the maximum power-conversion efficiency is $0.31 \%$. The power conversion efficiency achieved is about half of the efficiency achievable using the typical two-layer solar cell with the same organic materials. Yet, the device clearly shows the photovoltaic operation and the function of recycling incident ambient light.

Figure $7(\mathrm{~b})$ shows the $I-V$ characteristics of the solar cell (beneath the OLED) in dark and under the illumination of the OLED above (driven at $20 \mathrm{~mA} / \mathrm{cm}^{2}$, corresponding to $315 \mathrm{~cd} / \mathrm{m}^{2}$ observed outside the device). The solar cell exhibits clear photovoltaic response when the OLED is turned on. The photocurrent of the solar cell shows a linear relationship with the external OLED brightness, as shown in the inset of Fig. 7(b). When driving the OLED at $20 \mathrm{~mA} / \mathrm{cm}^{2}$ $\left(315 \mathrm{~cd} / \mathrm{m}^{2}\right)$, the solar cell gives an $I_{\mathrm{sc}}$ of $1.36 \mathrm{~mA} / \mathrm{cm}^{2}$, a $V_{\mathrm{oc}}$ of $0.43 \mathrm{~V}$, and a FF of $65.5 \%$, corresponding to the maximum power of $0.38 \mathrm{~mW}$ recycled from the OLED operation and a power recycling efficiency (output power of solar cell/electrical input power of OLED) of $0.26 \%$. This present recycling efficiency appears modest, yet one should notice that devices (both OLEDs and solar cells) used in this work are simply for the demonstration purpose and that there is plenty of room for further raising the recycling efficiency via using more efficient OLED and solar-cell structures. Overall, results of Figs. 7(a) and 7(b) clearly show that the solar cell integrated with the OLED not only recycles the photon energy incident from the environment, but also the photon energy generated internally from the OLED.

In summary, by integrating OLEDs with solar cells, ambient-light reflection as low as $1.4 \%$ (even superior to that achieved with polarizers) can be achieved without compromising the EL efficiency for high-contrast display applications. Furthermore, in such a configuration, the photon energies of both the incident ambient light and the portion of OLED emission not getting outside of the device can be recycled into useful electrical power via the photovoltaic action, instead of being wasted as in other reported contrastenhancement techniques. These features, we believe, shall make this present technique attractive for high-contrast display applications and portable/mobile electronics that are highly power-aware.

\section{References}

1 C. W. Tang and S. A. VanSlyke, Appl. Phys. Lett. 51, 913 (1987).

2 M.-H. Lu, M. S. Weaver, T. X. Zhou, M. Rothman, R. C. Kwong, M. Hack, and J. J. Brown, Appl. Phys. Lett. 81, 3921 (2002).

3 C.-C. Wu, P.-Y. Hsieh, C.-L. Lin, and H.-H. Chiang, Appl. Phys. Lett. 84, 3966 (2004).

4 L. S. Hung and J. Madathil, Adv. Mater. 13, 1787 (2001).

5 H. Aziz, Y.-F. Liew, H. M. Grandin, and Z. D. Popovic, Appl. Phys. Lett. 83, 186 (2003).

6 S. H. Li, H. Liem, C. W. Chen, E. H. Wu, Z. Xu, and Y. Yang, Appl. Phys. Lett. 86, 143514 (2005).

7 Z. Y. Xie and L. S. Hung, Appl. Phys. Lett. 84, 1207 (2004).

8 X. D. Feng, R. Khangura, and Z. H. Lu, Appl. Phys. Lett. 85, 497 (2004).

9 F. L. Wong, M. K. Fong, X. Jiang, C. S. Lee, and S. T. Lee, Thin Solid Films 446, 143 (2004).

10 C.-J. Yang, C.-L. Lin, C.-C. Wu, Y.-H. Yeh, C.-C. Cheng, Y.-H. Kuo, and T.-H. Chen, Appl. Phys. Lett. 87, 143507 (2005).

11 C. W. Tang, Appl. Phys. Lett. 48, 183 (1986).

12 L. D. Partain, Solar Cells and Their Applications (John Wiley \& Sons, Inc. 1995), pp. 165-177.

13 C.-W. Chen, Y.-J. Lu, C.-C. Wu, E. H.-S. Wu, C.-W. Chu, and Y. Yang, Appl. Phys. Lett. 87, 241121 (2005).

14 C.-I. Wu, C.-T. Lin, Y.-H. Chen, M.-H. Chen, Y.-J. Lu, and C.-C. Wu, Appl. Phys. Lett. 88, 152104 (2006).

15 X. Zhou, M. Pfeiffer, J. Blochwitz, A. Werner, A. Nollau, T. Fritz, and K. Leo, Appl. Phys. Lett. 78, 410 (2001).

16 H. Riel, S. Karg, T. Beierlein, B. Ruhstaller and W. Rieß, Appl. Phys. Lett. 82, 466 (2003).

17 C.-L. Lin, H.-W. Lin, and C.-C. Wu, Appl. Phys. Lett. 87, 021101 (2005) 